\title{
Actitudes lingüísticas de estudiantes universitarios hacia las lenguas quechua y español
}

\author{
Linguistic attitudes of university students towards \\ Quechua and Spanish languages
}

Óscar Esteban Roldán Rosales (iD Abelardo Rodolfo Campana Concha

Universidad Nacional Mayor de San Marcos. Lima, Perú

Recibido: 18/04/2021 Revisado: 19/04/2021 Aceptado: 03/05/2021 Publicado: 31/ 06/2021

\section{RESUMEN:}

Este estudio descriptivo correlacional tuvo como objetivo de la investigación analizar las actitudes lingüísticas que muestran los estudiantes de la Universidad Nacional "Santiago Antúnez de Mayolo" hacia el quechua y el español, para lo cual se utilizó la encuesta como técnica de recolección de datos, la cual fue aplicada a una muestra de 150 estudiantes, quienes tuvieron que responder a 12 preguntas en base a la variable de investigación. Los resultados señalaron que los estudiantes tienen diferentes opiniones sobre las lenguas quechua y español, no obstante que muestran una mayor valoración positiva hacia la lengua española. Asimismo, asignan distintos grados de uso, aunque es el español la lengua más preferida. Por ello, se concluye que los estudiantes otorgan actitudes favorables hacia el quechua y el español. Así, por lo que se refiere a las actitudes hacia el quechua se otorgan valoraciones positivas en el sentido de que esta lengua les proporciona mejores oportunidades de interrelación familiar y social, oportunidades laborales y eventualmente progreso económico, mientras que hacia el castellano priman las valoraciones en razón a los fines socioculturales, económicos y académicos. Además, la muestra investigada demostró una alta preferencia por el uso de la lengua española ya que esta lengua es la que predominantemente se utiliza en las comunicaciones diaria, además cuentan con un alto grado de conciencia y sensibilidad hacia la identidad cultural y lingüística del quechua. Palabras clave: Actitud lingüística, lengua, quechua, español, estudiante universitario, sociolingüística.

\section{ABSTRACT:}

This descriptive correlational study aimed to analyze the linguistic attitudes shown by the students of the National University "Santiago Antúnez de Mayolo" towards Quechua and Spanish, for which the survey was used as a data collection technique, the was applied to a sample of 150 students, who had to answer 12 questions based on the research variable. The results indicated that the students have different opinions about the Quechua and Spanish languages, although they show a higher positive assessment of the Spanish language. Likewise, they assign different degrees of use, although Spanish is the most preferred language. The- 
refore, it is concluded that students grant favorable attitudes towards Quechua and Spanish. Thus, with regard to attitudes towards Quechua, positive evaluations are given in the sense that this language provides them with better opportunities for family and social interrelation, job opportunities and eventually economic progress, while Spanish valuations prevail in reason for sociocultural, economic and academic purposes. In addition, the sample investigated showed a high preference for the use of the Spanish language since this language is the one that is predominantly used in daily communications, and they also have a high degree of awareness and sensitivity towards the cultural and linguistic identity of Quechua. Keywords: Linguistic attitude, language, Quechua, Spanish, university student, sociolinguistics.

\section{INTRODUCCIÓN}

El Perú es un país multiétnico, multilingüe y plurilingüe. Esta caracterización generalizada, sin embargo, ha llevado a varios autores a plantear diversos puntos de vista. Así por ejemplo. Caravedo (1996, 2001), menciona "la situación lingüística en el Perú actual se caracteriza por su alto grado de conflictividad, hecho que, según esta autora, se debe fundamentalmente al contacto o convivencia de lenguas o de variedades del español peruano. Es indudable, que este contacto lingüístico, se ha acrecentado con los movimientos demográficos que se han producido en nuestro país, especialmente, en la segunda mitad del siglo XX”.

En el marco de este contexto, particularmente, dentro del contacto entre las lenguas quechua y castellano en la región Ancash y de nuestro país, el fenómeno del manejo lingüístico, contribuye como aspecto importante y merece ser estudiado. Pues, los hablantes bilingües y no bilingües de estas lenguas no pueden ser ajenos a mostrar determinados comportamientos y valoraciones hacia dichas lenguas.

El comportamiento lingüístico, son maneras mostradas por los hablantes ante lenguas o variedades de lenguas que usan o conocen. Al respecto, Moreno Fernández (2009: 177) las define como "la manifestación de la actitud social de los individuos, distinguida por centrarse y referirse específicamente tanto a la lengua, o a cualquiera de sus variedades, como al uso que de ella se hace en la sociedad". Es innegable, que sobre el estudio de este tema, en la actualidad se conocen muchos aportes, principalmente en el ámbito internacional. Así, para efectos de nuestro estudio, a nivel de Hispanoamérica, resulta importante destacar los. Granda (1972), quien estudió respecto la lingüística en Puerto Rico, en el que utilizó una metodología un tanto distinta a la que ya tradicionalmente se maneja en la mayoría de los trabajos; Rubin (1974), menciona "análisis detallado de los diversos factores que intervienen en la conducta individual, describe la situación lingüística bilingüe del Paraguay en relación a los dos idiomas hablados en este país: español y guaraní, el análisis estuvo referido a las variables actitudes, estabilidad, uso, adquisición y grado de eficiencia de las lenguas en cuestión". Alvar (1986) presenta un amplio marco teórico al respecto del tema tratado; ofrece panorama sobre la realidad sociolingüística del medio, relacionando desde el punto de vista sociológico y psicológico del medio lingüístico.

Asimismo, en el país son importantes, por un 
lado, los aportes de Uta von Gleich (1989), quien en su investigación realizada con hablantes bilingües de Ayacucho, resalta hacia el castellano y quechua, establecidas por actitud lingüística practicado por diversos clases sociales; según Roldán (2008), el sociocultural influye en la actitud lingüística de gente bilingüe quechua-castellano hablante mayores de 35 años de edad del Callejón de Huaylas, además, estos pobladores presentan alto grado de valorar la lengua de origen. Falcón (2017), investigó sobre actitudes lingüísticas en pobladores indígenas bilingües, selva central del Perú, considerando dimensiones: afectiva, cognoscitiva y sociocultural, considerando: edad, sexo, estudios y procedencia, detalla los pobladores de comunidades yanesha y ashaninka, mayormente los jóvenes y muchos adultos muestran actitudes afectivamente negativas con alto grado de deslealtad lingüística hacia su lengua nativa; mientras que la población nomatsigenga, en su mayoría adultos y mayores, pondera la lengua castellana desde el punto de vista cognoscitivo, en cambio, desde la dimensión afectiva, manifiesta un fuerte lazo de identidad con la su lengua.

Cabe manifestar que dada la complejidad advertida en propuestas muestran los hablantes en actos de comunicación diaria, los propósitos, es importante mencionar todo hablante, aparte de su condición sociocultural, revela determinadas preferencias al lenguaje que utiliza, resultan de gran interés para su estudio. Pues, como sabemos, en el contexto peruano, los bilingües, en base formas de pensar, tienen posibilidades de evidenciar comportamientos como: lealtad o deslealtad, hacia las lenguas que conocen o usan; valorar de los hablantes variedad lingüística, presentando primordialmente estudios lingüísticos del momento.
El artículo tiene la finalidad dar conocer, desde punto sociolingüístico, los resultados, en estudiantes universitarios de pregrado. Dar respuesta a preocupaciones qué piensan la muestra sobre el quechua y castellano, identificar en contextos comunicativos, qué grados de valoraciones otorgan a dichas lenguas, en qué medida se deben usar estas lenguas en las diversas situaciones comunicativas de la universidad, entre otros aspectos relevantes en relación a este tema.

\section{MATERIALES Y MÉTODOS}

La presente publicación se sustenta en una investigación de tipo descriptiva, desde una perspectiva mentalista que hace referencia a la indagación del estado interno o mental del individuo hacia una situación sociolingüística concreta Moreno, 2009). Conforme a los objetivos, el presente estudio trata la actitud lingüística, en el marco del diseño no experimental de corte transaccional, sustentándose en estudio cualitativo con datos corroborados en una encuesta, aplicada a los participantes del estudio.

Los participantes de la investigación lo representan los estudiantes universitarios de pregrado, Escuela Profesional de Educación UNASAM, se delimitó la población para generalizar los resultados. Hernández, Fernández y Baptista (2010), mencionan "si se pretenden generalizar los resultados a una población, las muestras probabilísticas son esenciales. Además, los autores agregan que para el muestreo probabilístico se necesitan dos pasos: determinar el tamaño de la muestra y seleccionar los sujetos de forma aleatoria".

La población estuvo conformada por 510 sujetos, para determinar la muestra se utilizó el muestreo probabilístico, 
mediante la técnica aleatoria simple, seleccionándose150 estudiantes como muestra.

Se aplicó una encuesta, cuyo propósito fue valorar la opinión sobre el uso del idioma quechua y español. Se utilizó un cuestionario cuyo diseño estuvo estructurado en tres secciones alrededor de 12 preguntas. En la primera sección se indagaban acerca de algunos datos demográficos como (sexo, edad, lugar de nacimiento, etc.). En la segunda sección se plantearon preguntas que indagaban sobre el conocimiento y dominio de uso de las lenguas en cuestión, en los diversos contextos comunicativos, y en la tercera sección las preguntas planteadas indagaban acerca de sus preferencias, opinión y gusto personal de los estudiantes con respecto a las lenguas quechua y español.

\section{RESULTADOS Y DISCUSIÓN}

Los resultados que se presentan en este artículo forman parte de una investigación más amplia cuyo objetivo es analizar los datos en profundidad y desde una perspectiva cualitativa, no obstante, se presenta un avance del estudio, limitado en su alcance y desde una perspectiva cualitativa, permitiendo dar cuenta de los aspectos involucrados de la variable en estudiada.

Se analizó de los datos arrojados en el instrumento de recolección de información, los resultados se han organizado en cuatro partes: en primer lugar, se ubican algunos datos demográficos de los participantes; en segundo lugar, los aspectos cognitivos de las actitudes lingüísticas, en tercer lugar, los aspectos afectivos de las actitudes asociados a las dos lenguas evaluadas y, finalmente, los aspectos de las actitudes relacionados con las preferencias y opiniones sobre el quechua y español. Sobre las características demográficas de los participantes, estos datos fueron meramente informativos porque no constituyeron relevantes para la investigación. Sin embargo, cabe manifestar que, como ya se indicó, en este estudio participaron 150 estudiantes universitarios de pregrado, que estaban cursando distintas carreras de la Escuela Profesional de Educación, entre el primero y el décimo ciclos y cuya edad oscilaba entre los 17 y los 25 años. Asimismo, por su procedencia (zona sierra de Ancash) y permanencia en el lugar de estudios (Huaraz), los estudiantes seleccionados fueron monolingües (español) en un 44.5 $\%$ y bilingües (quechua y español) en un 55.5 $\%$, condición que puede explicarse debido a la información que nos brindaron los participantes en los ítems 1 y 2 del cuestionario.

En cuanto, al conocimiento y dominio del quechua y español, los ítems 3, 4 y 5, tratan sobre los dominios sobre uso de lengua quechua y español, relacionados las habilidades lingüísticas básicas, el 81\% de los estudiantes, respondieron que participan sin dificultad en la comunicación oral en español, 96\% afirman leen con facilidad y $83 \%$ escriben bien, uso del español manifestaron estudiantes dominio de oralidad y escritura siendo relevante. Referente al quechua, en cambio, los resultados dan cuenta que el $40 \%$ muestra dominio en la expresión oral y el 53\% está capacitado para la habilidad de la escucha. Por otro lado, en lo que respecta a las habilidades de leer y escribir el porcentaje de dominio se ve reducido grandemente, de tal modo que el $13.5 \%$ asegura que lee bien algún tipo de texto informativo mientras que solo el 3.9\% asevera que escribe bien en esta lengua.

Sobre las valoraciones sobre el uso de las lenguas quechua y español, en los ítems 6, 
7, 8 y 10; las apreciaciones de los estudiantes, otorgan la lengua quechua y española, conveniencias de uso en la vida familiar, social, profesional o laboral, así como al prestigio social y al grado de identificación, se advierte que la lengua quechua es la menos favorecida. Así, 39\% asevera la lengua quechua no usan en lo académico, 36\% manifiestan no usan en el ámbito laboral, el 29\%, $22 \%$, en el ámbito social y $8.5 \%$ asegura no utilizan en la vida familiar. Por lo tanto, estos datos revelan que para un porcentaje mayoritario de estudiantes la lengua quechua no es apropiada para ser usada en los distintos ámbitos de la vida diaria. Lo que hace suponer que la lengua española sí es la más apropiada para los fines comunicativos y de uso en estos diversos campos de la actividad humana.

Con respecto al prestigio que adquieren estas lenguas, nuestros encuestados manifiestan en un $75 \%$ que ambas lenguas se encuentran en iguales condiciones, el $14.5 \%$ considera que el quechua posee un rango inferior, mientras que el 9.5\% sostiene que la lengua nativa es la que posee un rango superior frente al castellano. Estos datos nuevamente ponen al quechua en condiciones inferiores de valoración en cuanto al rasgo de prestigio lingüístico.

En lo concerniente al grado de identificación de los estudiantes con las lenguas en cuestión, los datos recogidos nos muestran que alrededor del $58 \%$ se siente identificado como hablante de su lengua materna, vale decir con el quechua mientras que un $6 \%$ siente vergüenza por esta lengua que habla.

En suma, las respuestas revelan poca valoración de los participantes sobre el quechua para comunicarse, e identificarse con dicha lengua.
Con respecto a las preferencias sobre el uso del quechua y español, en los ítems 4, 9, 11 y 12 que exploran las preferencias y las opiniones de los estudiantes sobre las lenguas que hablan en la comunicación entre pares, 98\% manifiesta, utilizar la lengua española tanto en lugares públicos y $1.5 \%$ practica el idioma quechua. En la universidad, 99\% de los estudiantes se comunican en lengua castellana y el $1 \%$, en quechua. Además, en las reuniones sociales, el $98 \%$ se comunica en lengua española y solo el $1.5 \%$ se comunica en lengua quechua. De esta manera se advierte claramente la preferencia de uso del castellano y por lo tanto la predominancia sobre el quechua. Con respecto a la opinión recogida sobre los gustos para leer y escribir en la universidad, el 90\% de los encuestados manifiestan que lo harían en quechua y en castellano y el $10 \%$ asegura que lo haría únicamente en español. Asimismo, la opinión acerca de qué lengua les parece más bella, el 70\% asegura que la lengua quechua es más bella y el 30\% afirma que es la lengua española. Indudablemente, en esta respuesta encontramos una valoración positiva hacia el idioma quechua. De lo anterior se deduce que un alto porcentaje de los participantes son hablantes bilingües que tienen al quechua como lengua materna.

Los hallazgos recogidos constituyen una valiosa fuente de información para posibilitar, desde la voz de los participantes, un análisis de los aspectos que merezcan una revisión o que requieran una profundización en futuros estudios acerca de este tema. En este trabajo, en torno a las actitudes lingüísticas, los resultados ponen de manifiesto la importancia que los estudiantes conceden a las valoraciones y a las preferencias 
hacia las lenguas quechua y español; aunque dichas valoraciones sea asimétrica, cobra relevancia tomar en cuenta para atribuir las significaciones correspondientes.

En el marco de la dimensión cognitiva es importante destacar que existe una diferenciación significativa en el dominio de uso de las habilidades comunicativas orales y escritas en favor de la lengua española. A pesar de que el mayor porcentaje de los participantes son bilingües quechua-castellano hablantes, este hecho se explica debido a que en ellos hay una marcada influencia del contexto sociocultural determinado por el proceso de aprendizaje de dicha lengua a lo largo de los estudios escolares previos a la universidad, lo cual es comprensible por cuanto que esta diferenciación también servirá para establecer una marca de estatus sociocultural. Esto se confirma con lo manifestado por Roldán (2008), en el sentido de que las características socioculturales influyen fuertemente en las actitudes lingüísticas de los hablantes sean monolingües o bilingües. Desde la dimensión afectiva, los resultados revelan las actitudes positivas que favorecen a la lengua española, una evidencia importante que se advierte y que es necesario resaltar es la alta valoración que los participantes otorgan hacia la lengua castellana sin que esto signifique necesariamente una deslealtad hacia la lengua quechua. Este hecho es explicable en un contexto de contacto lingüístico diglósico castellano-quechua como es el caso específico de la ciudad de Huaraz, lugar de estancia de los estudiantes, donde encontramos que la lengua castellana goza de mayor prestigio y es la lengua preferida para el uso en las distintas situaciones comunicativas, incluyendo, indudablemente, el ambiente académico.
Continuando con esta dimensión, en relación a las categorías de prestigio lingüístico y al grado de identificación con las lenguas quechua y castellana, un alto porcentaje (75\%) de los participantes manifiesta que ambas lenguas poseen el mismo valor, se encuentran en las mismas condiciones; sin embargo, a pesar de que hay un porcentaje considerable que atribuye el valor de superior a la lengua quechua, no se puede soslayar que esta lengua sigue siendo desvalorizada. Asimismo, con respecto al grado de identificación con las lenguas, si bien es cierto que más del $50 \%$ se atribuye identificado con la lengua quechua, un porcentaje considerable manifiesta que siente vergüenza por su lengua nativa de lo cual se infiere que en este rubro también la lengua española adquiere mayor valoración, con lo que nuevamente es la lengua de mayor prestigio y que por lo tanto goza de un mejor estatus. Al respecto, de acuerdo con Utta von Gleich (1989), sostenemos que las características socioculturales además de los rasgos de identidad y prestigio influyen en las actitudes lingüísticas de los hablantes. En relación a las preferencias y opiniones, dimensión conativa, a la luz de los resultados establecemos claramente que la preferencia de uso en los distintos ambientes comunicativos es hacia la lengua castellana aun en los ámbitos cercanos a la vida diaria como es la vida familiar, comunal, laboral y aún más en el ámbito académico. Sin embargo, no se puede dejar de destacar que los participantes aspiran a que la lengua quechua sea también valorada funcionalmente al igual que el castellano y así adquiera el carácter instrumental en ambientes específicos como la universidad. Se infiere que esta aspiración se apoya en la apreciación y alta valoración que estos 
hablantes otorgan a la lengua quechua en el sentido de que esta es una lengua bella para expresar y recrear la cosmovisión de sus hablantes. A decir de Chávez "La cosmovisión quechua trasluce la concepción del mundo como respuesta del hombre, que intenta descifrar la realidad física del universo dentro del cual desarrolla su existencia” (Chávez, 2003, p. 41). Finalmente, es preciso señalar que, aun cuando los resultados mostrados son sumamente importantes para los propósitos de esta investigación, faltaría ampliar este tipo de estudios considerando otras variables que den cuenta de aspectos más completos y tal vez en mayor profundidad, las cuales no han sido abordadas en este trabajo como por ejemplo, cuáles son las causas que originan estas actitudes lingüísticas. Pues, como es obvio, siempre habrá algún otro motivo para emprender estudios acerca de este fenómeno sociolingüístico.

\section{CONCLUSIONES}

En concordancia con lo manifestado en los párrafos precedentes, a la luz de los resultados y análisis presentados en este estudio, se concluye que los estudiantes de pregrado de la Universidad Nacional Santiago Antúnez de

Mayolo manifiestan, alta preferencia del uso del idioma español. Asimismo, otorgan actitudes muy positivas hacia esta lengua que trae consigo el mejoramiento del estatus sociocultural de estos hablantes.

Sobre las actitudes lingüísticas vinculadas con las dimensiones cognitiva, afectiva y conativa se manifiestan de manera preferencial hacia la lengua española en los diversos ambientes comunicativos; sin embargo, no se puede soslayar que también evidencian una marcada preferencia y valoración hacia la lengua quechua, sobre todo, cuando se trata de atribuir rasgos de carácter sociocultural. A partir del aprovechamiento de la diversidad cultural y lingüística de nuestro país, es imperativo ampliar el estudio en la valoración de factores que causan y originan la conducta lingüística de otras lenguas, cuyos resultados apoyen implementar políticas educativas pertinentes en el empleo de lengua originaria como es el caso específico del quechua.

\section{REFERENCIAS BIBLIOGRÁFICAS}

Alvar, M. (1986). Hombre, etnia, estado. Gredos.

Caravedo, R. (1996). Perú. En M. Alvar (Ed.), Manual de dialectología hispánica: El español de América (153-168). Editorial Ariel.

Caravedo, R. (2001). Una interpretación geo social del español de América. Lexis, 25 (1-2), 51-57.

Chávez, A. (2003). La toponimia en la zona andina de Ancash. Con especial referencia al topónimo SHIQUI. Universidad Nacional Mayor de San Marcos.

De Granda, G. (1972). Transculturación e interferencia en el Puerto Rico contemporáneo. Cátedra.

Falcón C., P. (2017). Identidades y actitudes lingüísticas en contextos interculturales en comunidades bilingües amazónicas [Tesis Magisterial, Universidad Nacional Mayor de San Marcos]. 
Hernández, R., Fernández, C. y Baptista, P. (2010). Metodología de la investigación. McGraw-Hill.

Moreno, F. (2009) Principios de sociolingüistica y sociología del lenguaje. Ariel S.A.

Roldán R. (2008). Actitudes lingüísticas en los pobladores bilingües del callejón de Huaylas. Revista Aporte Santiaguino, 5, 57-71.

Rubín, J. (1974). Bilingüismo nacional en el Paraguay. Instituto Indigenista Interamericano.

Von Gleich, U. (1989). Actitudes lingüisticas entre hablantes bilingües en Ayacucho. Temas de lingüistica aplicada. CONCYTEC. 